Kakuya Kitagawa

Hajime Sakuma

Motonori Nagata

Shigeo Okuda

Masaharu Hirano

Akihiro Tanimoto

Masaki Matsusako

Joao A. C. Lima

Sachio Kuribayashi

Kan Takeda

\section{Diagnostic accuracy of stress myocardial perfusion MRI and late gadolinium-enhanced MRI for detecting flow-limiting coronary artery disease: a multicenter study}

Received: 15 February 2008

Revised: 1 May 2008

Accepted: 9 June 2008

Published online: 24 July 2008

(C) The Author(s) 2008

K. Kitagawa $\cdot$ H. Sakuma ( $\bowtie) \cdot$

M. Nagata $\cdot$ K. Takeda

Department of Radiology,

Mie University Hospital,

2-174 Edobashi,

Tsu, Mie, 514-8507, Japan

e-mail: sakuma@clin.medic.mie-u.ac.jp

Tel.: +81-59-2315029

Fax: +81-59-2328066

S. Okuda · A. Tanimoto ·

S. Kuribayashi

Department of Radiology,

Keio University School of Medicine,

Tokyo, Japan

\section{Hirano}

Department of Internal Medicine,

Tokyo Medical University,

Tokyo, Japan

\author{
M. Matsusako \\ Department of Radiology, \\ St. Luke's International Hospital, \\ Tokyo, Japan \\ K. Kitagawa · J. A. C. Lima \\ Division of Cardiology, \\ Department of Medicine, \\ Johns Hopkins University, \\ Baltimore, MD, USA
}

\begin{abstract}
The aim of this study was to determine the diagnostic performance of stress and rest perfusion magnetic resonance imaging (MRI) and late gadolinium-enhanced (LGE) MRI for identifying patients with obstructive coronary artery disease (CAD). A total of 50 patients with suspected CAD underwent stress-rest perfusion MRI, followed by LGE MRI with a $1.5-\mathrm{T}$ system. Stress-rest perfusion MRI resulted in an area under the receiveroperating characteristic curve (AUC)
\end{abstract}

of 0.92 for observer 1 and 0.84 for observer 2 with sensitivity and specificity of $89 \%(32 / 36)$ and $79 \%$ $(11 / 14)$ by observer $1,83 \%(30 / 36)$ and $71 \%(10 / 14)$ by observer 2 , respectively, showing a moderate interobserver agreement (Cohen's k= 0.49 ). While combination of stressrest perfusion and LGE MRI did not result in improved accuracy for the prediction of flow-limiting obstructive CAD (AUC 0.81 for observer 1 and 0.80 for observer 2), the sensitivity was increased to $92 \%$ in both observers with a substantial interobserver agreement $(\kappa=0.70)$. Stress-rest myocardial perfusion MRI is an accurate diagnostic test for identifying patients with obstructive CAD.

Keywords Coronary artery disease . Multicenter trial $\cdot$ Myocardial perfusion - Magnetic resonance imaging · Pharmacological stress

\section{Introduction}

In spite of the recent progress in noninvasive visualization of coronary artery trees using multidetector computed tomography and magnetic resonance imaging (MRI) [1-4], myocardial perfusion imaging is remaining as an important part of the assessment of patients with ischaemic heart disease. Myocardial perfusion is evaluated by single photon emission computed tomography in most patients today; however, this technique exposes patients to radiation and its diagnostic accuracy is sometimes limited by relatively low spatial resolution and artifacts from photon scatter and tissue attenuation.

First-pass contrast-enhanced MRI of the myocardium has emerged as a noninvasive method that can allow accurate assessment of myocardial ischaemia caused by flow-limiting stenosis without radiation [5-8]. Sensitivities of $81-93 \%$ and specificities of $68-90 \%$ have been reported for stress first-pass perfusion MRI in the detection of clinically significant coronary artery disease (CAD) as defined at coronary angiography [9-17]. However, to the best of out knowledge, there are only three multicenter 
studies demonstrating the diagnostic performance of firstpass perfusion MRI for the detection of CAD [12-14]. Moreover, none of the three studies included late gadolinium-enhanced (LGE) MRI in the analysis. The purpose of this multicenter study was to prospectively determine the diagnostic accuracy of stress-rest perfusion MRI and LGE MRI in detecting flow-limiting obstructive CAD using conventional coronary angiography as a reference standard.

\section{Materials and methods}

\section{Patients}

This prospective multicenter trial was performed at three clinical sites, with ethics committee approval in each site. Written informed consent was obtained from all patients. A total of 50 patients with known or suspected CAD who met the following inclusion and exclusion criteria were enrolled. The number of patients from each clinical site enrolled was 29,10 , and 11 , respectively. The study group included 36 men and 14 women aged 41-83 years (mean age 65.4 \pm 9.4 ). Patient demographics were summarized in Table 1. Patients were included in the study if (1) they were scheduled for cardiac catheterization with suspect of current obstructive coronary artery stenosis based on clinical symptom and/or positive stress test and (2) there was no contraindication to contrast-enhanced MRI. Patients were excluded if they were medically unstable, had contraindications for adenosine triphosphate (ATP) stress (i.e., second- or third-degree A-V block, sick sinus syndrome, known hypersensitivity to ATP). Patients with clinical myocardial infarction by history or medical record

Table 1 Patient demographics (PCI percutaneous coronary intervention)

\begin{tabular}{ll}
\hline Variables & Number of patients \\
\hline Patient characteristics: & \\
Sex: male/female & $36 / 14(72 \% / 28 \%)$ \\
Age (years) & $65 \pm 9$ \\
Range (years) & $41-83$ \\
Medical history: & \\
Arterial hypertension & $26 / 50(52 \%)$ \\
Diabetes mellitus & $23 / 50(46 \%)$ \\
Hyperlipidemia & $29 / 50(58 \%)$ \\
Smoker/prior history of smoking & $21 / 50(42 \%)$ \\
Patients with previous PCI & $4 / 50(8 \%)$ \\
Coronary status: & \\
Obstructive CAD/no obstructive CAD & $36 / 14(72 \% / 28 \%)$ \\
Single-vessel & $12 / 36(33 \%)$ \\
Double-vessel & $16 / 36(44 \%)$ \\
Triple-vessel & $8 / 36(22 \%)$ \\
\hline
\end{tabular}

and those who had previously undergone coronary artery bypass graft surgery were excluded.

\section{MRI protocol}

MRI was performed using 1.5-T systems of the same manufacturer. Three institutions had a different model $(\mathrm{CV} / \mathrm{i}$, Twinspeed, Echospeed, GE Medical Systems, Milwaukee, Wis., USA), but all of them had similar imaging capability with the same software version and pulse sequences. Systems were equipped with gradient strength of 33$40 \mathrm{mT} / \mathrm{m}$; slew rate of $120-150 \mathrm{~T} / \mathrm{m} / \mathrm{s}$ and a four-element phased-array coil. Patients were advised to withdraw caffeine intake for $24 \mathrm{~h}$ prior to the test. A 20-gauge catheter was inserted into an antecubital vein for contrast agent injection and a 22-gauge catheter into an antecubital vein of the other arm for pharmacologic stress agent administration.

First-pass contrast-enhanced MR images were obtained during pharmacologic stress and in the resting state with an ECG-gated saturation-recovery steady-state free precession (SSFP) perfusion sequence [18]. The sequence consisted of a non-section-selective $90^{\circ}$ saturation pulse and a single-shot SSFP image acquisition with sequential k-space sampling. The following parameter settings were used in all institutions: 3.0/1.2/250 [repetition time (ms)/echo time $(\mathrm{ms}) /$ inversion time (ms)]; inversion time represents time between saturation pulse to the center of acquisition window); flip angle $45^{\circ}$; band width of $64 \mathrm{kHz} ; 340-380 \mathrm{~mm}$ field of view; $128 \times 96$ matrix; 8 -mm thick sections; a phase FOV factor of 0.75 ; and a voxel size of $72 \mathrm{~mm}^{3}(3 \mathrm{~mm} \times 3 \mathrm{~mm} \times 8 \mathrm{~mm})$. Four to six short-axis images of the left ventricle were obtained for every other heartbeat for approximately $1 \mathrm{~min}$. For hyperemic perfusion imaging, contrast injection and image acquisition began 3 min after the initiation of ATP infusion $(140 \mu \mathrm{g} / \mathrm{kg} / \mathrm{min})[19,20]$. Patients were monitored for possible adverse event caused by ATP infusion, such as persistent or symptomatic AV block, severe hypotension and dyspnea. As soon as dynamic image acquisition started, $0.05 \mathrm{mmol} / \mathrm{kg}$ gadopentetate dimeglumine (Magnevist; Schering, Berlin, Germany) was injected into the antecubital vein with an MRI-compatible power injector at a rate of $4 \mathrm{ml} / \mathrm{s}$, followed by a 20-ml saline flush. The ATP infusion was then stopped $4 \mathrm{~min}$ after it was initiated. Approximately $15 \mathrm{~min}$ after the cessation of ATP infusion, resting (non-hyperemic) first-pass perfusion MR images were acquired with the same imaging parameters and dose of contrast agent. During the first-pass imaging, patients were requested to hold their breath as long as they could, then make shallow breathing thereafter so that respiratory artifacts are minimized. Cardiac functional MRI using cine SSFP sequence was also performed between stress and rest perfusion MRI, but was not evaluated in this study.

Subsequently, $0.05 \mathrm{mmol}$ gadopentetate dimeglumine per kilogram of body weight was administered in order to obtain cumulative dose of $0.15 \mathrm{mmol} / \mathrm{kg}$, which was optimized for 
late gadolinium-enhancement imaging. After $10 \mathrm{~min}$, late enhancement images were acquired in short-axis sections by using an ECG-gated inversion-recovery prepared twodimensional (2D) fast gradient-echo MR sequence in order to detect possible subclinical scars that otherwise remain unrecognized. Parameters for inversion-recovery gradientecho sequences were as follows: 5.3/1.3/230-270 [repetition time(ms)/echo time (ms)/inversion time (ms)]; flip angle, $25^{\circ}$; field of view, $340-380 \mathrm{~mm}$; matrix, $256 \times 128$; and section thickness, $5 \mathrm{~mm}$. The inversion time was adjusted to minimize the signal from normal myocardium by acquiring several test images with different inversion times.

\section{MRI analysis}

All patient studies were collected and anonymized. Two radiologists independently reviewed all the image datasets without knowing patient history, including age and sex, other imaging and laboratory study results. Prestudy experience of observer 1 and 2 in cardiovascular MRI was equivalent to level 3 ( $>300$ cases, 1 year) and $2(>150$ cases, 3 months), respectively, based on the guideline compiled by the Society the American College of Cardiology Task Force [21]. Stress and rest perfusion images were displayed side by side and visually evaluated by manually paging the images. In addition, LGE images were presented to assess the presence of myocardial scar. The observers recorded the presence or absence of stressinduced hypoperfusion using the AHA 16-segment model with a scale of 1-5: $1=$ definitely normal, $2=$ probably normal, 3 = possibly abnormal, 4 = probably abnormal, and $5=$ definitely abnormal. The most apical segment in the AHA 17-segment model was excluded from the analysis, since evaluation of apex was impossible on the short axis images. Analysis of the LGE MRI was also performed based on the 16-segment model and the same five-point scale used for perfusion MRI analysis.

Before reviewing cases for this study, these observers completed a training session together, which consisted of a review of ten MR studies including stress-rest perfusion and LGE and their coronary catheterization results. These cases were not included in the current patient population. Through the training session, they shared the following interpretation criteria. Stress-induced hypoperfusion was considered present if a focal region of myocardium showing diminished contrast enhancement was present during stress that was not observed at rest. If similar hypointensity was observed both during stress and at rest, it was considered to be an artifact or scar, and absence of stress-induced hypoperfusion was recorded. Therefore, LGE without perfusion abnormality was interpreted as negative for myocardial ischaemia. If hypointensity area during stress was larger than that at rest, it was interpreted as stress-induced ischemia with partial artifact or scar, and presence of stress-induced hypoperfusion was recorded.
The observers also agreed on how to differentiate true perfusion defect from subendocardial artifact. The differentiation was performed on the basis of their signal intensities, duration and distribution. Typically, signal intensities of artifacts are lower than that of the same region before contrast injection and appear at the time of contrast agent inflow into the left ventricle lasting only for a few images in the perfusion series [22-24]. In regard to distribution, circular hypointensity regions throughout the subendocardium of the left ventricle were considered to be more suggestive of artifact, whereas perfusion defects characterized by subendocardial predominance and segmental distribution of coronary artery were considered to be indicative of true perfusion defect.

\section{Conventional coronary angiography}

Conventional X-ray coronary angiography was considered as the reference standard for the evaluation of coronary artery stenosis. Conventional coronary angiography was performed within 14 days of the contrast-enhanced MR study at the same center that MR study was performed. Images were then transferred to one of the three centers and analysed by an observer with 20 years of experience in coronary angiography. The observer was blinded to both clinical as well as MR images and evaluated all epicardial coronary artery segments with diameter of $2 \mathrm{~mm}$ or greater including branches by using a workstation (Advantage CRS and QCA package; GE Medical Systems). The presence or absence of luminal diameter reduction of $50 \%$ or greater on coronary angiography was recorded in the three major coronary arteries. In order to correlate the coronary artery distribution on coronary angiography with myocardial perfusion abnormality on MRI, the AHA classification cross-sectional myocardial segments used for MRI analysis were assigned to three major coronary artery territories (left anterior descending artery, circumflex artery, and right coronary artery) based on the coronary artery distribution demonstrated by coronary angiography.

\section{Statistical analysis}

Statistical analyses were performed with a commercially available statistical analysis package, SPSS 11.5 J (SPSS, Chicago, Ill., USA). Continuous variables are expressed as mean \pm SD. The efficacy of the contrast-enhanced MR study for each reader was evaluated using receiveroperating characteristic curve (ROC) analyses based on the five-point scale visual assessment. Sensitivity, specificity, positive and negative predictive values, and accuracy with the corresponding $95 \%$ confidence interval (CI) were calculated using binary assessment as positive (score $\geq 3$ ) versus negative (score $<3$ ). Cohen's $k$ statistic was used to assess for interobserver agreement on the binary assess- 
ments. Combined assessment of first-pass perfusion MRI and LGE MRI was performed based on the higher score of the two modalities.

\section{Results}

Quantitative coronary angiography

The prevalence of obstructive CAD defined on lesions $>50 \%$ stenosis was $72 \%$ (36/50 patients). Twelve patients had single-vessel disease, 16 patients had double-vessel disease, and eight patients had triple-vessel disease.

Stress-rest perfusion MRI for detecting obstructive CAD

First-pass contrast-enhanced MR images were of adequate quality for image interpretation in all patients (Fig. 1). No


Fig. 1a-d Comparison between MRI and conventional coronary angiography in a 65-year-old patient. a, b Short-axis perfusion MR series acquired with a saturation-recovery SSFP sequence (3.0/1.2; field of view, $340 \mathrm{~mm}$; matrix, $128 \times 96$; flip angle, $45^{\circ}$ ) (a) during ATP stress and (b) at rest. Images of the same slice were shown to demonstrate contrast wash-in and wash-out. Perfusion deficits (arrows) were observed in the anterolateral wall and inferior wall of the left ventricle during stress. c Short-axis LGE MR images of the patient acquired with an inversion-recovery spoiled gradient echo sequence (5.3/1.3; field of view, $340 \mathrm{~mm}$; matrix, $256 \times 128$, serious adverse event was reported during ATP stress. Subendocardial dark rim artifact was observed in $40(80 \%)$ patients on at least one short-axis slice of stress-rest perfusion MRI. These artifacts were differentiated from true perfusion defect based on their characteristic signal behavior and distribution, as discussed above. The sensitivity of stress-rest first-pass perfusion MRI in the identification of patients with flow-limiting stenosis of at least one coronary artery was $89 \%(32 / 36 ; 95 \%$ CI 76,96$)$ and $83 \%(30 / 36 ; 95 \%$ CI 69,93$)$ by observer 1 and 2 , respectively. The specificity of MRI in detecting patients with flow-limiting stenosis in the coronary artery was $79 \%$ $(11 / 14 ; 95 \%$ CI 53,94$)$ by observer 1 and $71 \%(10 / 14 ; 95 \%$ CI 46,90 ) by observer 2 (Table 2). Cohen's $k$ statistic showed a moderate interobserver agreement $(\kappa=0.49)$. The area under the ROC curve (AUC) in this patient-based analysis was $0.92(95 \% \mathrm{CI} 0.83,0.99)$ for observer 1 and 0.84 (95\% CI $0.73,0.96)$ for observer 2 (Fig. 2). Sensitivities by observer 1 and 2 were $83 \%(10 / 12 ; 95 \%$ CI 56,96$)$ and $75 \%(9 / 12 ; 95 \%$ CI 47,92$), 88 \%(14 / 16$;
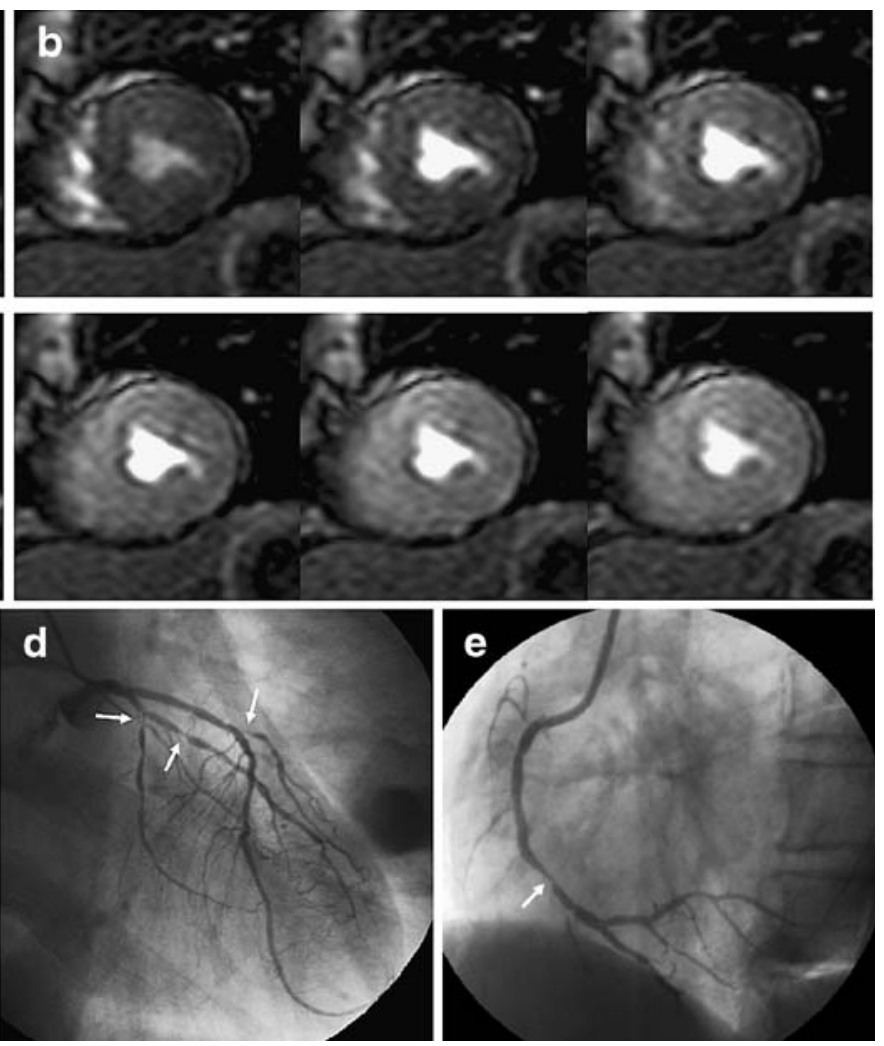

flip angle, $25^{\circ}$ ). Two focal hyperenhancements were observed in the anterior wall and inferolateral wall. Note the much smaller size of the late hyperenhancement compared to perfusion deficit on stress first-pass perfusion MR images. d Right anterior oblique conventional angiogram demonstrated multiple highgrade stenoses in the diagonal branch of the left anterior descending artery and left circumflex artery (arrows). e Left anterior oblique conventional angiogram displayed right coronary artery with a high grade stenosis (arrow) 
Table 2 Diagnostic performance of stress-rest perfusion MRI for detecting patients with flow-limiting coronary artery stenosis

\begin{tabular}{ll} 
Observer & \\
\hline 1
\end{tabular}

Sensitivity and 95\% CI (\%) 89 (32/36); 76,96 83 (30/36); 69,93 Specificity and 95\% CI (\%) 79 (11/14); 53,94 71 (10/14); 46,90 Positive predictive value $91(32 / 35) ; 79,98 \quad 88(30 / 34) ; 74,96$ and $95 \% \mathrm{CI}(\%)$

Negative predictive value $73(11 / 15) ; 48,90 \quad 63(10 / 16) ; 38,83$ and $95 \% \mathrm{CI}(\%)$

Accuracy and 95\% CI (\%) 86 (43/50); 75,94 80 (40/50); 67,89

95\% CI 66, 97) and 81\% (13/16;95\% CI 58, 94), and $100 \%(8 / 8 ; 95 \%$ CI 74,100$)$ and $100 \%(8 / 8 ; 95 \%$ CI 74 , $100)$, respectively, for the detection of single-, double-, and triple-vessel disease.

The sensitivity and specificity of stress first-pass contrast-enhanced MRI for predicting flow-limiting stenosis in the individual coronary artery were $81 \%(55 / 68 ; 95 \% \mathrm{CI}$ $70,89)$ and $83 \%(68 / 82 ; 95 \%$ CI 74,90$)$ by observer 1 , $72 \%(49 / 68 ; 95 \%$ CI 61,82$)$ and $87 \%(71 / 82 ; 95 \%$ CI 78 , 93) by observer 2 with a substantial interobserver agreement $(\kappa=0.61)$ (Table 3$)$. The area under the ROC curve in this vessel-based analysis was 0.83 (95\% CI 0.77 , $0.90)$ for observer 1 and $0.82(95 \%$ CI $0.74,0.89)$ for observer 2 (Fig. 3).

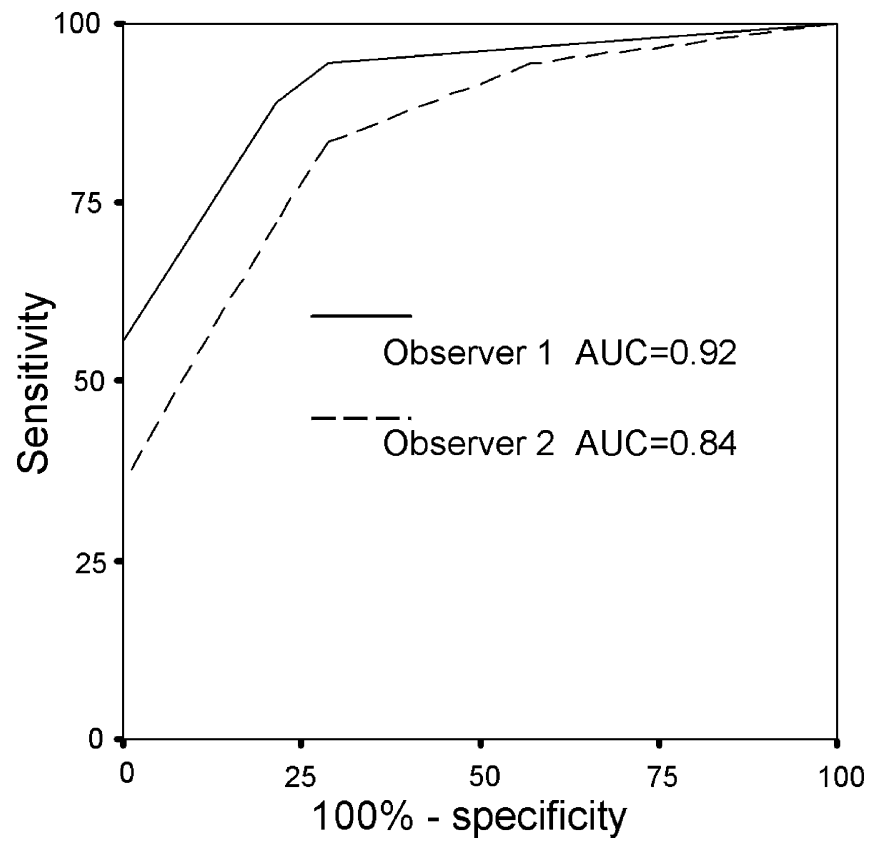

Fig. 2 ROC curves of stress-rest perfusion MRI for predicting patients with flow-limiting coronary artery disease. The AUC was 0.92 for observer 1 and 0.84 for observer 2
Table 3 Diagnostic performance of stress-rest perfusion MRI for detecting flow-limiting stenosis in individual coronary arteries

\begin{tabular}{ll}
\hline Observer \\
\cline { 2 - 2 } 1 & 2 \\
\hline
\end{tabular}

Sensitivity and 95\% CI (\%) 81 (55/68); 70,89 72 (49/68); 61,82

Specificity and 95\% CI (\%) 83 (68/82); 74,90 87 (71/82); 78,93

Positive predictive value $\quad 80(55 / 69) ; 69,88 \quad 82(49 / 60) ; 71,90$ and $95 \% \mathrm{CI}(\%)$

Negative predictive value $84(68 / 81) ; 75,91 \quad 79$ (71/90); 70,86 and $95 \% \mathrm{CI}(\%)$

Accuracy and 95\% CI (\%) 82 (123/150); 75,88 80 (120/150); 73,86

\section{LGE MRI for detecting obstructive CAD}

Small focal areas of LGE were observed in 24 (48\%) and 26 $(52 \%)$ of the 50 patients by observer 1 and 2 , respectively, in spite of the exclusion of patients with clinical myocardial infarction from the study population. When presence of LGE was used for predicting flow-limiting coronary artery stenosis irrespective of perfusion MRI findings, its sensitivity and specificity for detecting patients with obstructive CAD was $56 \%(20 / 36 ; 95 \%$ CI 39,71$)$ and $71 \%(10 / 14 ; 95 \%$ CI 46,90$)$ by observer 1 , and $61 \%(22 / 36 ; 95 \%$ CI 45,76$)$ and $71 \%(10 / 14 ; 95 \%$ CI 46,90$)$ by observer 2 , respectively, with a substantial interobserver agreement $(\kappa=0.68)$. The AUC was $0.64(95 \%$ CI $0.46,0.81)$ for observer 1 and 0.67

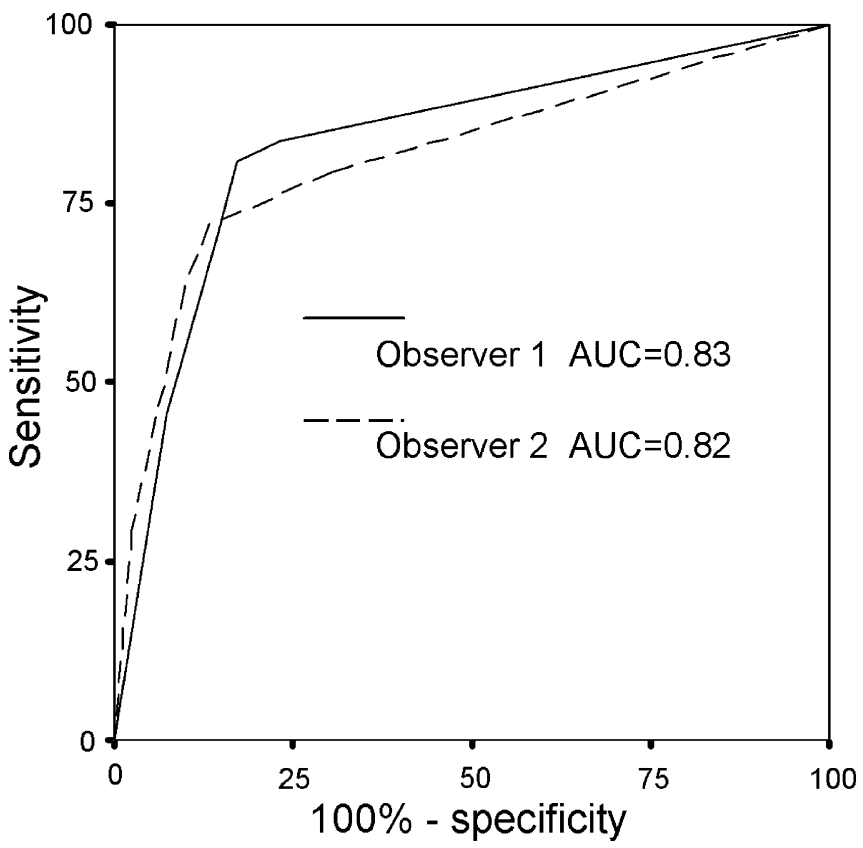

Fig. 3 ROC curves of stress-rest perfusion MRI for detecting flowlimiting stenosis in the individual coronary artery. The AUC was 0.83 for observer 1 and 0.82 for observer 2 
(95\% CI $0.51,0.84)$ for observer 2 in the patient based analysis. At the vessel level analysis, sensitivity and specificity of LGE MRI was $32 \%(22 / 68 ; 95 \%$ CI 22,44$)$ and $93 \%(76 / 82 ; 95 \% \mathrm{CI} 86,97)$ by observer 1 , and $35 \%$ $(24 / 68 ; 95 \%$ CI 25,47$)$ and $93 \%(76 / 82 ; 95 \%$ CI 86,97$)$ by observer 2, with a substantial interobserver agreement $(\mathrm{K}=$ $0.74)$. The ROC curve analysis at the vessel level showed an AUC of $0.62(95 \%$ CI $0.53,0.72)$ and 0.64 (95\% CI 0.55 , 0.73 ) for observer 1 and 2, respectively.

Combination of stress-rest perfusion MRI and LGE MRI yielded sensitivity and specificity of $92 \%(33 / 36$; $95 \%$ CI $79,98)$ and $57 \%(8 / 14 ; 95 \%$ CI 32,80$)$ by observer $1,92 \%$ $(33 / 36 ; 95 \%$ CI 79,98$)$ and $50 \%(7 / 14 ; 95 \%$ CI 26,74$)$ by observer 2, respectively, for identifying patients with obstructive CAD with a substantial interobserver agreement $(\kappa=0.70)$ (Table 4). Area under the ROC curve was $0.81(95 \%$ CI $0.66,0.96)$ and $0.80(95 \%$ CI $0.64,0.95)$ for observer 1 and 2, respectively (Fig. 4). At the vessel level analysis, its sensitivity and specificity was $82 \%(56 / 68$; $95 \%$ CI 72,90$)$ and $78 \%(64 / 82 ; 95 \%$ CI 68,86$)$ by observer $1,75 \%(51 / 68 ; 95 \%$ CI 64,84$)$ and $79 \%(65 / 82$; $95 \%$ CI 70,87 ) by observer 2 , respectively, with a substantial interobserver agreement $(K=0.65)$ (Table 5). Area under the ROC curve for vessel level analysis was $0.82(95 \%$ CI $0.75,0.89)$ and $0.80(95 \%$ CI $0.73,0.88)$ for observer 1 and 2, respectively (Fig. 5).

\section{Discussion}

This study demonstrates (1) that stress-rest first-pass perfusion MRI using SSFP technique and ATP is an accurate diagnostic test for the detection of flow-limiting $\mathrm{CAD}$, and (2) combining presence of scar on LGE MRI can improve sensitivity and interobserver agreement, but does not improve the overall diagnostic performance of MRI

Table 4 Diagnostic performance of combination of stress-rest perfusion MRI and LGE MRI for detecting patients with flowlimiting coronary artery stenosis

\begin{tabular}{lll}
\hline & \multicolumn{2}{l}{ Observer } \\
\cline { 2 - 3 } & 1 & 2 \\
\hline $\begin{array}{l}\text { Sensitivity and 95\% CI (\%) } \\
\text { Specificity and 95\% CI (\%) }\end{array}$ & $57(8 / 14) ; 32,80$ & $50(7 / 14) ; 26,74$ \\
$\begin{array}{l}\text { Positive predictive value } \\
\text { and 95\% CI (\%) }\end{array}$ & $85(33 / 39) ; 71,93$ & $83(33 / 40) ; 69,92$ \\
$\begin{array}{l}\text { Negative predictive value } \\
\text { and 95\% CI (\%) }\end{array}$ & $73(8 / 11) ; 43,92$ & $70(7 / 10) ; 39,91$ \\
\begin{tabular}{l} 
Accuracy and 95\% CI (\%) \\
\hline
\end{tabular} & $82(41 / 50) ; 70,91$ & $80(40 / 50) ; 67,89$ \\
\hline
\end{tabular}

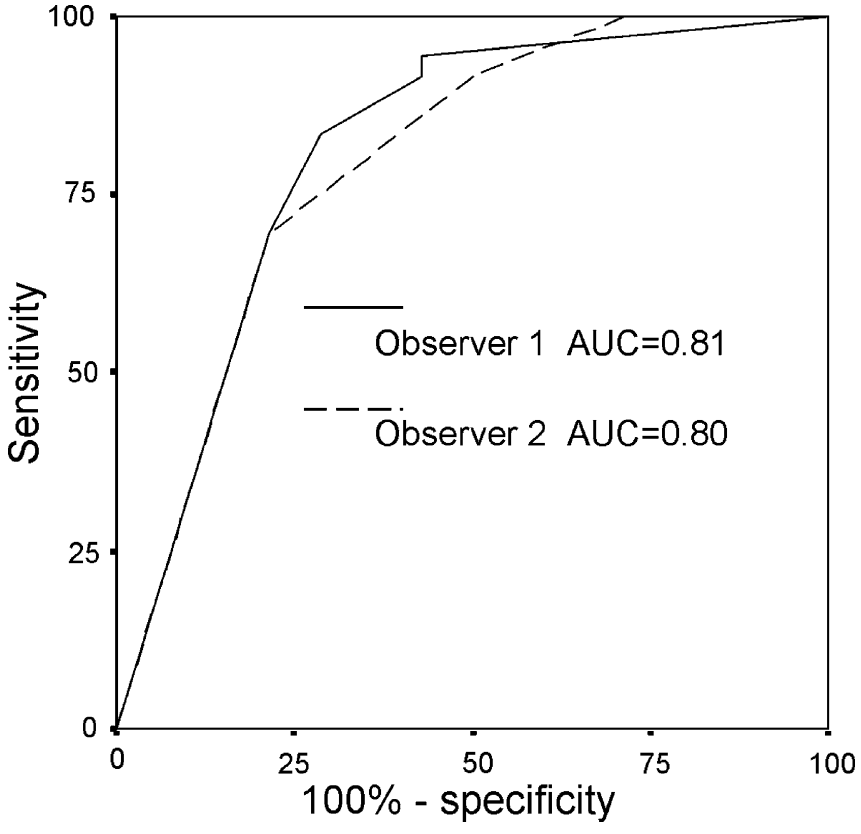

Fig. 4 ROC curves of combination of stress-rest perfusion MRI and LGE MRI for predicting patients with flow-limiting coronary artery disease. The AUC was 0.81 for observer 1 and 0.80 for observer 2

study when luminal narrowing on coronary angiography is used as a reference method.

Diagnostic performance of stress-rest perfusion MRI

Recent studies showed that first-pass contrast-enhanced MRI with vasodilator stress allows for the accurate detection of hemodynamically significant coronary artery stenosis $[9-13,15,16]$. However, to our knowledge, only

Table 5 Diagnostic performance stress-rest perfusion MRI and LGE MRI for detecting flow-limiting stenosis in individual coronary arteries

\begin{tabular}{lll}
\hline & \multicolumn{2}{l}{ Observer } \\
\cline { 2 - 3 } & 1 & 2 \\
\hline $\begin{array}{l}\text { Sensitivity and 95\% CI (\%) } \\
\text { Specificity and 95\% CI (\%) }\end{array}$ & $78(56 / 68) ; 72,90$ & $75(51 / 68) ; 64,84$ \\
$\begin{array}{l}\text { Positive predictive value } \\
\text { and 95\% CI (\%) }\end{array}$ & $76(56 / 74) ; 65,84$ & $75(51 / 68) ; 64,84$ \\
$\begin{array}{l}\text { Negative predictive value } \\
\text { and 95\% CI (\%) }\end{array}$ & $84(64 / 76) ; 75,91$ & $79(65 / 82) ; 70,87$ \\
Accuracy and 95\% CI (\%) & $80(120 / 150) ; 73,86$ & $77(116 / 150) ; 70,83$ \\
\hline
\end{tabular}




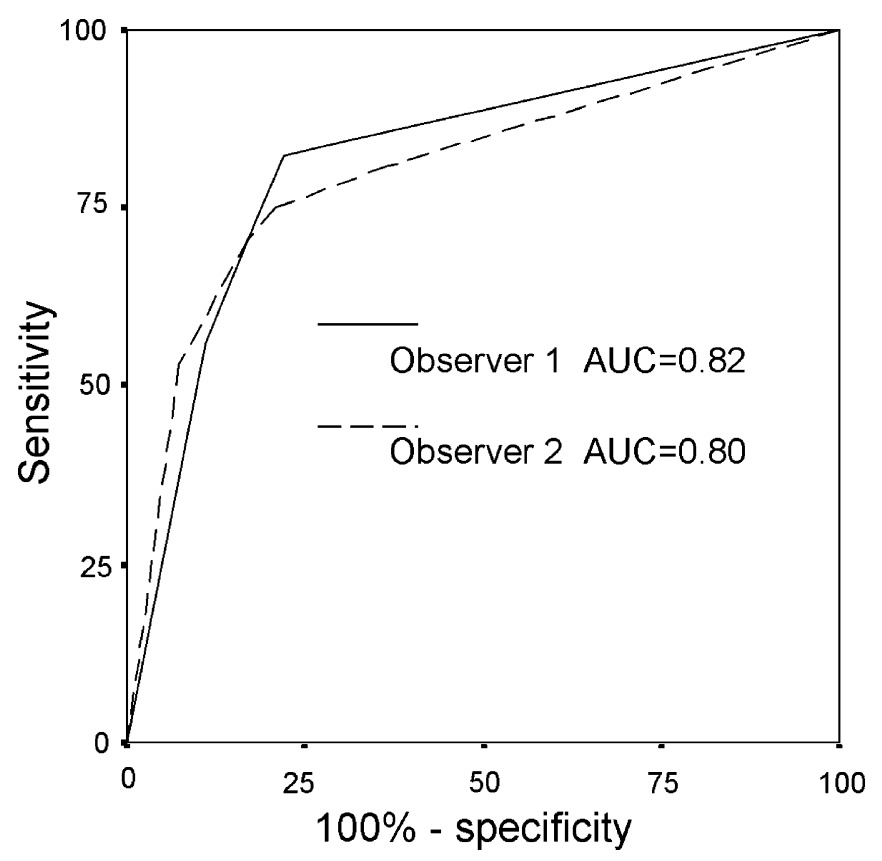

Fig. 5 ROC curves of combination of stress-rest perfusion MRI and LGE MRI for detecting flow-limiting stenosis in the individual coronary artery. The AUC was 0.82 for observer 1 and 0.80 for observer 2

three multicenter studies are available. Giang et al. [13] monitored wash-in kinetics of contrast medium using a hybrid echo-planar pulse sequence in 94 patients (up to 27 patients per subgroup receiving the same contrast dose) at three clinical centers. By assessing the upslope of the timeintensity curve, single hyperemic perfusion MRI showed a sensitivity of $93 \%$ and specificity of $75 \%$. Wolff et al. [12] obtained first-pass contrast-enhanced MR images in 99 patients (up to 26 patients per subgroup receiving the same contrast dose) at three clinical sites by using a segmented echo-planar imaging pulse sequence with a notched saturation pulse. By visual assessment of stress and rest perfusion MRI, the sensitivity and specificity of first-pass contrast-enhanced MRI in detecting patients with significant coronary stenosis were $93 \%$ and $75 \%$, respectively. The largest multicenter perfusion MRI study published so far is MR-IMPACT, which included 234 patients (up to 50 patients per subgroup receiving the same contrast dose) from 18 centeres [14]. By visual assessment, diagnostic performance of perfusion MRI in 42 patients who received optimal contrast dose showed similar performance as SPECT (AUC 0.86 for MRI vs 0.75 for SPECT) [14]. In the current study, perfusion MRI yields sensitivities and specificities of $89 \%$ and $79 \%$ (AUC 0.92 ) by an observer with CMR training level of 3 , and $83 \%$ and $71 \%$ (AUC 0.84) by an observer with CMR training level of 2 for the detection of patients with obstructive CAD. We employed saturation-recovery SSFP sequence for MR perfusion imaging in this study since this technique has been shown to have better signal-to-noise and contrast-to-noise ratio compared with spoiled gradient-echo and segmented echo-planar techniques [25]. Although SSFP perfusion is considered more susceptible to dark-rim artifacts, use of SSFP sequence resulted in at least no appreciable deterioration in diagnostic performance in our study cohort in comparison with previous reports.

\section{LGE MRI in the detection of obstructive CAD}

LGE images obtained with inversion-recovery prepared MR sequence permits the detection of small myocardial scars [26]. In the current study, small areas of late enhancement were observed in about a half of the study population in spite of the exclusion of patients with clinical myocardial infarction. The incidence of LGE in our study is not surprising given the high prevalence of CAD in this population sample. As a matter of fact, a recent populationbased study in Sweden showed that $19.8 \%$ of randomly selected 70-year-old subjects had subclinical myocardial infarct reflected as focal area of LGE [27].

Several studies have demonstrated that combining LGE MRI with stress-rest perfusion MRI can improve the sensitivity of MRI for obstructive CAD without deteriorating the specificity when only obvious myocardial infarction (i.e., LGE consistent with a coronary distribution) were considered [28, 29]. However, correlating the presence of LGE with flow-limiting obstructive CAD can be inappropriate since microinfarcts may occur without coronary artery narrowing as a result of plaque rupture/ ulceration and subsequent washout of the lipid pool with or without additional thrombus formation into the microcirculation $[30,31]$. As a result, the scar can be atypical for myocardial infarction. In fact, many of the areas of LGE in our study were small focal lesions rather than those showing typical appearance of myocardial infarction. And inclusion of these small focal LGE seems to be the reason why combination of stress-rest perfusion MRI and LGE MRI resulted in deterioration of specificity in our study. Nevertheless, combined assessment of stress-rest perfusion MRI and LGE MRI seems to be valuable. By adding LGE MRI, the sensitivity was increased to $92 \%$ in both of the two observers, reducing the chance of overlooking patients with obstructive CAD. In addition, substantial interobserver agreement was achieved with a combination of perfusion and LGE MRI $(\kappa=0.70)$ compared with moderate agreement with perfusion MRI alone $(\kappa=0.49)$, indicating that combined assessment can be less subjective and more independent of the observers' experience. Reduced specificity resulting from the combination of LGE MRI should not be interpreted as reduced diagnostic 
value because detection of unrecognized myocardial scar is valuable in the management of patients with $C A D$, since presence of unrecognized scar tissue has incremental prognostic impact beyond clinical, angiographic, and functional predictors [32].

\section{Limitations}

There are several limitations in this study. Firstly, the number of the patients is relatively small. Reliable estimation of specificity and negative predictive value at the patient level was not possible due to the relatively small number of patient population and the high prevalence of $\mathrm{CAD}$, and further investigation is required. Secondly, the morphological assessments of CAD by quantitative coronary angiography evaluated by a single observer as the standard of reference for perfusion MRI can be a limitation because myocardial perfusion MRI reflects the physiologic severity of coronary artery stenosis rather than its morphology. Third limitation in this study is that presence and absence of perfusion deficits were determined solely by visual assessment. Quantitative analysis of myocardial perfusion MRI potentially enhances the diagnostic performance of first-pass contrast-enhanced MRI.

\section{Conclusions}

This multicenter study demonstrated that stress-rest myocardial perfusion MRI acquired with a SSFP sequence and ATP has high sensitivity, specificity and a moderate interobserver agreement for identifying patients with obstructive CAD. Combination of LGE MRI is of diagnostic value since sensitivity and interobserver agreement of stress-rest perfusion MRI can be improved. Further investigation is required to determine the specificity and negative predictive value of stress-rest perfusion MRI, LGE MRI and combination of the two.

Open Access This article is distributed under the terms of the Creative Commons Attribution Noncommercial License which permits any noncommercial use, distribution, and reproduction in any medium, provided the original author(s) and source are credited.

\section{References}

1. Herzog C, Zwerner PL, Doll JR et al (2007) Significant coronary artery stenosis: comparison on per-patient and per-vessel or per-segment basis at 64section CT angiography. Radiology 244:112-120

2. Sakuma H, Ichikawa Y, Chino S et al (2006) Detection of coronary artery stenosis with whole-heart coronary magnetic resonance angiography. J Am Coll Cardiol 48:1946-1950

3. Leber AW, Johnson T, Becker A et al (2007) Diagnostic accuracy of dualsource multi-slice CT-coronary angiography in patients with an intermediate pretest likelihood for coronary artery disease. Eur Heart J 28:2354-2360

4. Nassenstein K, Waltering KU, Kelle S et al (2008) Magnetic resonance coronary angiography with vasovisttrade mark: in-vivo T(1) estimation to improve image quality of navigator and breath-hold techniques. Eur Radiol 18:103-109

5. Al-Saadi N, Nagel E, Gross M et al (2000) Noninvasive detection of myocardial ischemia from perfusion reserve based on cardiovascular magnetic resonance. Circulation 101:1379-1383
6. Klocke FJ, Simonetti OP, Judd RM et al (2001) Limits of detection of regional differences in vasodilated flow in viable myocardium by first-pass magnetic resonance perfusion imaging. Circulation 104:2412-2416

7. Panting JR, Gatehouse PD, Yang GZ et al (2002) Abnormal subendocardial perfusion in cardiac syndrome $X$ detected by cardiovascular magnetic resonance imaging. N Engl J Med 346:1948-1953

8. Ingkanisorn WP, Kwong RY, Bohme NS et al (2006) Prognosis of negative adenosine stress magnetic resonance in patients presenting to an emergency department with chest pain. J Am Coll Cardiol 47:1427-1432

9. Ishida N, Sakuma H, Motoyasu M et al (2003) Noninfarcted myocardium: correlation between dynamic first-pass contrast-enhanced myocardial MR imaging and quantitative coronary angiography. Radiology 229:209-216

10. Schwitter J, Nanz D, Kneifel S et al (2001) Assessment of myocardial perfusion in coronary artery disease by magnetic resonance: a comparison with positron emission tomography and coronary angiography. Circulation 103:2230-2235
11. Nagel E, Klein C, Paetsch I et al (2003) Magnetic resonance perfusion measurements for the noninvasive detection of coronary artery disease. Circulation 108:432-437

12. Wolff SD, Schwitter J, Coulden R et al (2004) Myocardial first-pass perfusion magnetic resonance imaging: a multicenter dose-ranging study. Circulation 110:732-737

13. Giang TH, Nanz D, Coulden R et al (2004) Detection of coronary artery disease by magnetic resonance myocardial perfusion imaging with various contrast medium doses: first European multi-centre experience. Eur Heart J 25:1657-1665

14. Schwitter J, Wacker CM, van Rossum AC et al (2008) MR-IMPACT: comparison of perfusion-cardiac magnetic resonance with single-photon emission computed tomography for the detection of coronary artery disease in a multicentre, multivendor, randomized trial. Eur Heart J 29:480-489

15. Sakuma H, Suzawa N, Ichikawa Y et al (2005) Diagnostic accuracy of stress first-pass contrast-enhanced myocardial perfusion MRI compared with stress myocardial perfusion scintigraphy. AJR Am J Roentgenol 185:95-102 
16. Meyer C, Strach K, Thomas D et al (2008) High-resolution myocardial stress perfusion at $3 \mathrm{~T}$ in patients with suspected coronary artery disease. Eur Radiol 18:226-233

17. Nandalur KR, Dwamena BA, Choudhri AF et al (2007) Diagnostic performance of stress cardiac magnetic resonance imaging in the detection of coronary artery disease: a meta-analysis. J Am Coll Cardiol 50:1343-1353

18. Foo TK, Wu KC, Azevedo CF et al (2004) Partial fourier steady-state free precession (FIESTA) first-pass perfusion with improved image quality and efficient spatial coverage. Proc Int Soc Magn Reson Med 11:1

19. Jeremias A, Filardo SD, Whitbourn RJ et al (2000) Effects of intravenous and intracoronary adenosine 5'-triphosphate as compared with adenosine on coronary flow and pressure dynamics. Circulation 101:318-323

20. Miyagawa M, Kumano S, Sekiya M et al (1995) Thallium-201 myocardial tomography with intravenous infusion of adenosine triphosphate in diagnosis of coronary artery disease. J Am Coll Cardiol 26:1196-1201
21. Pohost GM, Kim RJ, Kramer CM et al (2006) Task Force 12: training in advanced cardiovascular imaging (cardiovascular magnetic resonance [CMR]): endorsed by the Society for Cardiovascular Magnetic Resonance. J Am Coll Cardiol 47:910-914

22. Schreiber WG, Schmitt M, Kalden P et al (2002) Dynamic contrast-enhanced myocardial perfusion imaging using saturation-prepared TrueFISP. J Magn Reson Imaging 16:641-652

23. Arai AE (2000) Magnetic resonance first-pass myocardial perfusion imaging. Top Magn Reson Imaging 11:383398

24. Strach K, Meyer C, Thomas D et al (2007) High-resolution myocardial perfusion imaging at $3 \mathrm{~T}$ : comparison to $1.5 \mathrm{~T}$ in healthy volunteers. Eur Radiol 17:1829-1835

25. Wang Y, Moin K, Akinboboye $\mathrm{O}$ et al (2005) Myocardial first pass perfusion: steady-state free precession versus spoiled gradient echo and segmented echo planar imaging. Magn Reson Med 54:1123-1129

26. Wagner A, Mahrholdt H, Holly TA et al (2003) Contrast-enhanced MRI and routine single photon emission computed tomography (SPECT) perfusion imaging for detection of subendocardial myocardial infarcts: an imaging study. Lancet 361:374-379
27. Barbier CE, Bjerner T, Johansson L et al (2006) Myocardial scars more frequent than expected: magnetic resonance imaging detects potential risk group. J Am Coll Cardiol 48:765-771

28. Klem I, Heitner JF, Shah DJ et al (2006) Improved detection of coronary artery disease by stress perfusion cardiovascular magnetic resonance with the use of delayed enhancement infarction imaging. J Am Coll Cardiol 47:1630-1638

29. Cury RC, Cattani CA, Gabure LA et al (2006) Diagnostic performance of stress perfusion and delayed-enhancement MR imaging in patients with coronary artery disease. Radiology 240:39-45

30. Erbel R, Heusch G (1999) Coronary microembolization-its role in acute coronary syndromes and interventions. Herz 24:558-575

31. Heusch G, Schulz R, Haude M et al (2004) Coronary microembolization. J Mol Cell Cardiol 37:23-31

32. Kwong RY, Chan AK, Brown KA et al (2006) Impact of unrecognized myocardial scar detected by cardiac magnetic resonance imaging on event-free survival in patients presenting with signs or symptoms of coronary artery disease. Circulation 113:2733-2743 